\title{
RETRACTION
}

\section{DNA sequencing using electrical conductance measurements of a DNA polymerase}

Yu-Shiun Chen, Chia-Hui Lee, Meng-Yen Hung, Hsu-An Pan, Jin-Chern Chiou and G. Steven Huang

Nature Nanotechnology 8, 452-458 (2013); published online 5 May 2013; corrected after print 11 July 2013 and 28 August 2013 ; retracted after print 3 June 2015.

Significant concerns were raised about the validity of the data reported in this work shortly after publication. After an internal inquiry, a formal investigation was launched at the National Chiao Tung University, which focused on the reproducibility of the data. The results of the work could not be reproduced in a reasonable timeframe, and the authors could not provide the investigating committee with a complete set of raw data for the original experiments. The authors Y.-S. C., J.-C. C. and G. S. H. have therefore agreed to retract the manuscript; C.-H. L., M.-Y. H. and H.-A. P. did not respond to the journal's attempts to contact them about this retraction. 